\title{
A new RREQ message forwarding technique based on Bayesian probability theory
}

\author{
Venetis Kanakaris*, David L Ndzi, Kyriakos Ovaliadis and Yanyan Yang
}

\begin{abstract}
The flooding method, which is used by many mobile ad-hoc routing protocols, is a process in which a route request packet (RREQ) is broadcasted from a source node to other nodes in the network. This often results in unnecessary re-transmissions, causing packet collisions and congestion in the network, a phenomenon called broadcast storm. This article presents firstly the impact of a different message forwarding probability on the RREQ and secondly a RREQ message forwarding scheme which is implemented on Ad-hoc On-Demand Distance Vector Routing (AODV) routing protocol, a Bayesian probability based the AODV extended version based on a modified version of Bayesian probability (AODV_EXT_BP) that reduces routing overheads, by calculating the probability with respect to the neighbour density as well as the posterior probability. The performance of the AODV_EXT_BP is compared to that of extended version of AODV (AODV_EXT), AODV, Destination Sequenced Distance Vector, dynamic source routing and Optimized Link State Routing protocols and the simulation results show that the AODV_EXT_BP protocol achieves better results in all sectors.
\end{abstract}

Keywords: Mobile ad-hoc network, Routing message overhead, Route discovery, Broadcast, Bayesian probability, Flooding, Power-aware routing, Routing protocols, AODV, AODV_EXT, AODV_EXT_BP, DSDV, DSR, OLSR

\section{Introduction}

An ad-hoc network is a promising technology which can be applied in an extensive number of areas ranging from environmental monitoring to disaster management. Furthermore, ad-hoc networks can be implemented in application of sensors for process automation in a diversity of industrial applications. Events such as earthquakes can often serve to illustrate the weakness of centrally managed networks and the importance of research and development in ad-hoc networks such as mobile ad-hoc networks (MANETs), where a centralized connectivity is not needed. MANET is a wireless network which consists of mobile nodes with no pre-determined infrastructure. One of the major restrictions of ad-hoc network systems is the energy availability and continuous reduction in the size of devices which means that power reduction cannot simply be improved with big battery systems [1]. Apart from the operation of onboard electronics, power consumption depends on numerous processes and overheads required to maintain connectivity.

\footnotetext{
* Correspondence: venetis.kanakaris@port.ac.uk

School of Engineering, University of Portsmouth, Anglesea Road, Portsmouth PO1 3DJ, UK
}

\section{黑 Springer}

There are numerous recommended solutions to deal with connectivity problems and power limitation in adhoc networks. Such techniques comprise development of hardware, routing algorithms, protocols and battery technology or power management systems $[2,3]$. There are researchers that have suggested the development of an optimized hardware which can be used in applications based on data rates [4]. There is a proposal in $[5,6]$ to adapt energy control to applications where the voltage, and therefore processing speed and power, can be reduced for non-time sensitive applications. Other methods that have been proposed in [7] are aimed at avoiding network partitioning by controlling power consumptions of critical link nodes. Recently, many probabilistic approaches have been proposed in [8-11] with the aim to alleviate the flooding phenomenon and solve the broadcast storm problem. The authors have already proposed a probability-based algorithm in [12] which is an improved version of Ad-hoc OnDemand Distance Vector Routing (AODV), the AODV_EXT, which reduces energy consumption by $3 \%$. In this article, we propose a modification of AODV, the AODV_EXT_BP, protocol which improves the energy and data transmission 
efficiency of the network by using the Bayesian probability theory and give better results compared to AODV_EXT.

In general, ad-hoc wireless networks broadcast packets to the whole network as a means of transmitting information from one node to the other in the network [13]. Broadcasting in MANETs is not only an essential action for unicast routing protocols in mobile scenarios, but also a unique process of a number of multicast routing protocols. A variety of unicast, multicast, and geocast protocols uses the broadcasting method in order to provide the important control and route establishing functionality. Broadcasting a packet to the whole network has a wide range of applications in MANETs. Consequently, improving the process of broadcasting will result in savings in several adhoc applications.

Flooding is the simplest technique used by source nodes to broadcast packets to the adjacent nodes [14]. Each neighbour node receiving the packet for the first time rebroadcasts it ensuring forward propagation from the source node until every node in the network has received and transmitted the broadcast packet exactly once.

The broadcasting protocols can be classified into two main categories, namely deterministic and probabilistic. The probabilistic approach usually offers a simple solution in which every node that receives a broadcast packet has a predefined probability of forwarding the message [15]. But this approach does not ensure full network coverage. On the other hand, the deterministic approach can provide full coverage and can be further categorized into two categories, namely location information and neighbour set based.

In MANETs, the routing task is delivered through network nodes which act as both routers and end points in the network. In order for a route to a specific destination node to be discovered, existing on-demand routing protocols use a simple flooding mechanism whereby a route request packet (RREQ) originating from a source node is broadcasted without exception to all nodes in the network [16]. This can result to considerable redundant retransmissions, causing congestion and packet collisions in the network.

The implication of broadcast routing messages on network performance is presented in this article. The following protocols have been studied and their performances in simulated networks are analysed: dynamic source routing (DSR), AODV, Destination Sequenced Distance Vector (DSDV) routing and Optimized Link State Routing (OLSR). These protocols have widely been used and cited in literature [17].

This article is organized as follows: "Routing protocols" section describes the protocols that will be evaluated in this article. The routing procedure of AODV is also described. "The modification of AODV using different RREQ mechanism message forwarding probability scheme" and "Simulation and metrics of a different message forwarding
Table 1 Definition of the different message forwarding probability

\begin{tabular}{ll}
\hline$P_{t x}$ & Power consumed during forwarding a route control message \\
\hline$P_{r x}$ & Power consumed during receiving a route control message \\
$P_{\text {total }}$ & $\begin{array}{l}\text { The sum of total remaining power of the neighbouring nodes } \\
\text { node }\end{array}$ \\
$P_{i}$ & The sum of total initial powers of the $n$ neighbours \\
$P_{s}$ & The total power of all nodes \\
$n$ & Number of nodes in the neighbourhood \\
\hline
\end{tabular}

probability scheme on the RREQ mechanism of AODV protocol" sections present the modification and the results of the AODV behaviour when using different message forwarding probability implemented on the RREQ mechanism, respectively. "The modification of AODV RREQ mechanism using Bayesian probability approach" section presents a description of the proposed modification to AODV as well as the Bayesian probability theory. In "Simulation and metrics of AODV RREQ mechanism using the Bayesian approach" section, the simulated scenario is described together with the settings, network configurations and the parameters that have been used in order to evaluate the performance of the protocols. The results of the simulation and discussions are provided in "Results and discussions" section which is followed by the conclusions.

\section{Routing protocols}

In DSDV [18] protocol, there is an exchange of messages between mobile nodes within range. Routing updates may

\begin{tabular}{ll}
$\begin{array}{l}\text { Table } 2 \text { Simulation Parameters of AODV to evaluate } \\
\text { different RREQ message forwarding probabilities }\end{array}$ \\
\hline The protocol & $\begin{array}{l}\text { Probabilistic RREQ message } \\
\text { forwarding protocol }\end{array}$ \\
\hline Topographical area & $800 \times 800 \mathrm{~m}^{2}$ \\
Number of nodes & $10,20,30,40,50,60,70,80,90$, \\
& 100 \\
Mobility & $1-30 \mathrm{~m} / \mathrm{s}$ \\
Probability & $0.10-1.0$ (step 0.1) \\
Radio-propagation model & TwoRayGround \\
Network interface type & WirelessPhy \\
MAC type & $802 \_11$ \\
Interface queue type & DropTail/PriQueue \\
Antenna model & OmniAntenna \\
Total simulation time & $100 \mathrm{~s}$ \\
Transport/traffic type & CBR over UDP \\
Tx power of the nodes & $0.1819 \mathrm{~W}$ \\
Rx power of the nodes & $0.0501 \mathrm{~W}$ \\
Idle power of the nodes & $0.0350 \mathrm{~W}$ \\
Initial energy of the nodes & $1000.0 \mathrm{~J}$ \\
Scenario simulation repetitions & 10 \\
\hline
\end{tabular}




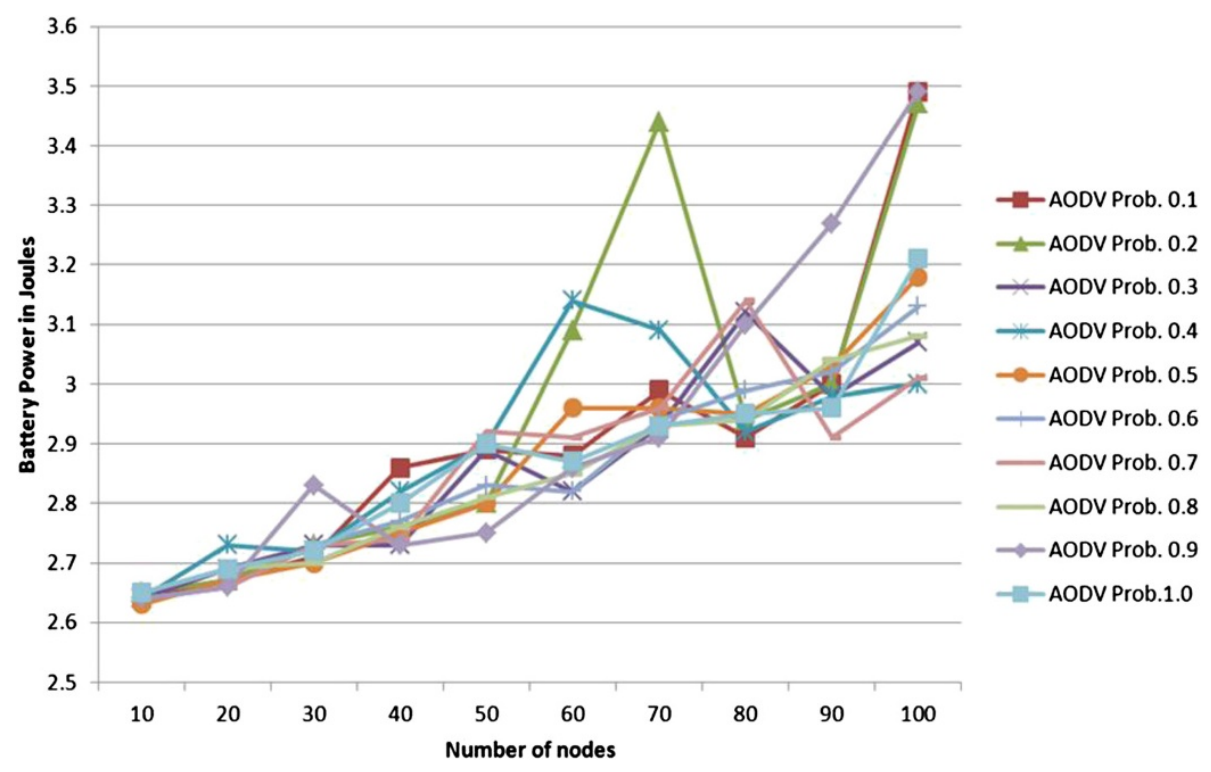

Figure 1 The average consumed power.

be enabled or routine. The update process starts when routing information from one of the adjacent nodes forces a change in the routing table. If there is a packet which the route to its destination is unknown, it is cached whilst routing queries are sent out. The packets are cached until route-replies are received from the destination node. The buffer has a specific size and time limitation for caching packets beyond which packets are dropped. When the route to the destination is known the packets are routed directly. In a case that the destination node is not found, the packets are forwarded to the default target node which is the routing agent.

In DSR protocol, the routing agent controls every data packet for source-route information [19]. The packets are then forwarded according to the routing information. In case, it cannot find any routing information in the packet, it offers the source route if route is known. When the destination is not known it caches the packet and broadcasts route queries. The routing query is initially sent to all the adjacent nodes and it is always enabled by a data packet

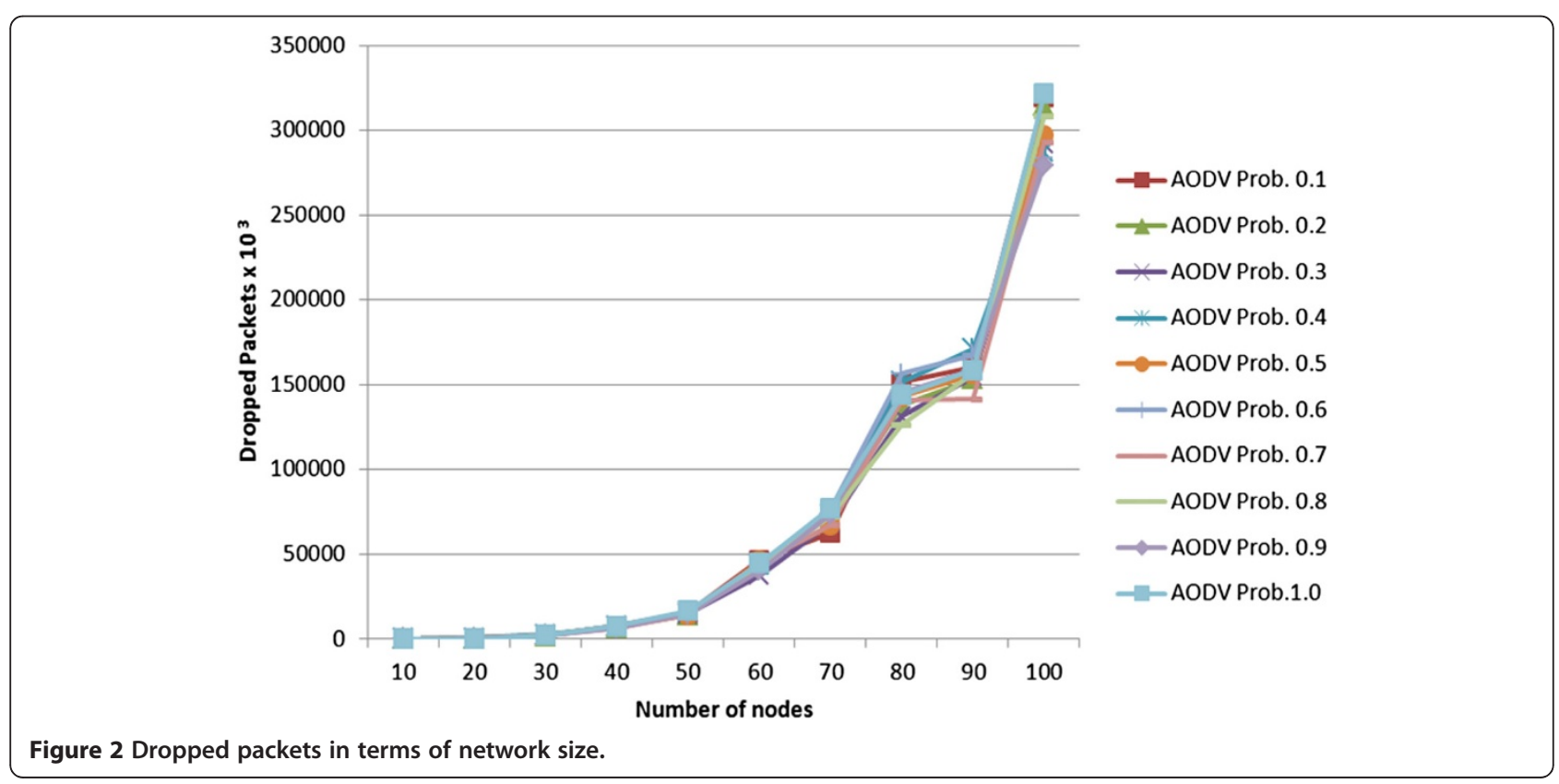




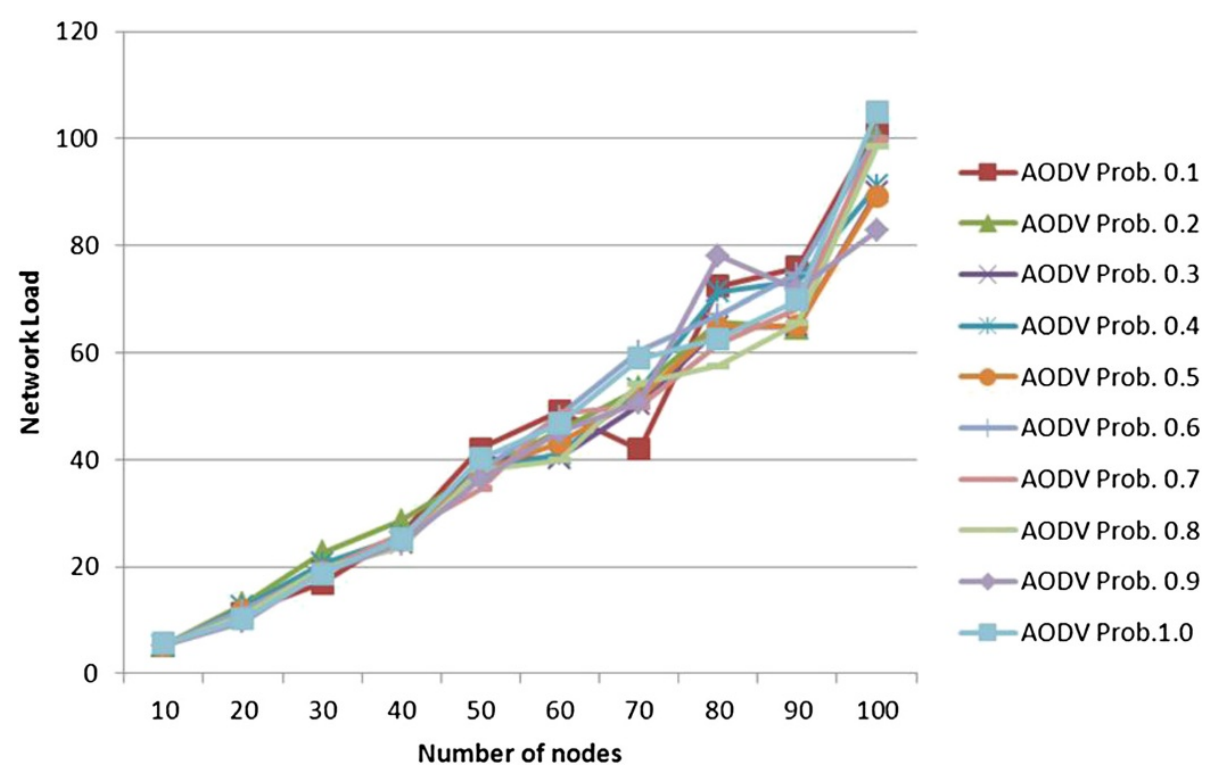

Figure 3 MAC Load against the number of nodes.

which has no route information about its destination. Route-replies are sent back if the information regarding the route to the destination is needed.

The OLSR [20] is a routing protocol in which the nodes are aware of all the valid routes. The OLSR protocol uses the flooding method in order to inform all active nodes in the network when there is a topology change. The OLSR reduces the possible overhead in the network by using the multi-point relays (MPR). The main concept of MPR [21] is to reduce the number of duplicate retransmissions when a broadcast packet is forwarded. By this method, the number of retransmissions is confined to a small group of nearby nodes, instead of using all the neighbours. This group of nodes is kept as small as possible by selecting the nodes which cover (in terms of one-hop radio range) the same network region as the complete set of nearby nodes. The OLSR routing protocol has two types of control

\section{Table 3 Definition of AODV_EXT_BP parameters}

\begin{tabular}{|c|c|}
\hline$n$ & The total nodes in the network \\
\hline$F_{i}$ & Any node $F_{i} i=1,2, \ldots, n$ that receives the RREQ message \\
\hline$P_{i}$ & Packet forwarding probability derived from neighbour node count \\
\hline$d$ & $\begin{array}{l}\text { Minimum node density or minimum expected neighbours - if the } \\
\text { node density at a forwarding node } F_{j} \text { is less than this, then that } \\
\text { node will forward the RREQ message to avoid the probability of } \\
\text { path failure at that location. This means that the nodes will } \\
\text { forward the packet without any further condition checking. In this } \\
\text { study } d \text { is set to } 5\end{array}$ \\
\hline r & $\begin{array}{l}\text { Random number (between } 0 \text { and } 1 \text { ). This is used to generate } \\
\text { varying conditions in the network. By this variable we are trying } \\
\text { to avoid flooding in the RREQ mechanism as it compares each } \\
\text { time to } P_{i} \text { which must be smaller than } 1(100 \%)\end{array}$ \\
\hline
\end{tabular}

messages, namely Topology Control (TC) and Hello. TC messages are used for sending information regarding the adjacent nodes which contains the MPR selector list, instead of Hello messages which are used in order to discover information about the link status and the host's neighbours. The OLSR protocol has a drawback due to the fact that every host periodically transmits the updated topology information to the whole network thus

Table 4 Simulation parameters and configuration of AODV, AODV_EXT, AODV_EXT_BP, OLSR, DSDV, DSR

\begin{tabular}{ll}
\hline Routing protocols & $\begin{array}{l}\text { AODV, AODV_EXT_BP, AODV_BP, } \\
\text { OLSR, DSDV, DSR }\end{array}$ \\
\hline Topographical area & $800 \times 800 \mathrm{~m}^{2}$ \\
Number of nodes & $10,20,30,40,50,60,70,80,90,100$ \\
Mobility & $1-30 \mathrm{~m} / \mathrm{s}$ \\
Channel type & Wireless channel \\
Radio-propagation model & TwoRayGround \\
Network interface type & WirelessPhy \\
MAC type & $802 \_11$ \\
Interface queue type & DropTail/PriQueue \\
Antenna model & OmniAntenna \\
Max packet in Queue & 50 \\
Transport/traffic type & CBR over UDP \\
TxPower of the nodes & $0.1819 \mathrm{~W}$ \\
RxPower of the nodes & $0.0501 \mathrm{~W}$ \\
IdlePower of the nodes & $0.0350 \mathrm{~W}$ \\
Initial energy of the nodes & $1000.0 \mathrm{~J}$ \\
Scenario simulation repetitions & 10 \\
\hline
\end{tabular}


increasing bandwidth usage. But this problem is solved by using the MPR, which forwards only the messages regarding the topology of the network.

The AODV protocol is a mix of DSDV and DSR protocols [22]. It uses the hop-by-hop routing sequence numbers and beacons of DSDV and keeps the basic routediscovery and route-maintenance mechanism of DSR. When a node needs to know a route to a specific destination, it generates an RREQ. The RREQ packet is forwarded by intermediate nodes which also create a reverse route from the destination. When the request reaches a node with a route to the destination node it also creates a Route Reply (RREP) which contains the number of hops that are required to reach the destination. The intermediate nodes that take part in forwarding, reply to the source node and create a forward route to destination. This route created from each node from source to destination is a hop-by-hop state and not the entire route as in source routing.

\section{The modification of AODV using different RREQ mechanism message forwarding probability scheme} In this article, we propose a modification to AODV protocol to use a probability-based message forwarding scheme in the RREQ mechanism. In the original AODV protocol, in the RREQ mechanism, 100\% (probability of 1.0) all intermediate nodes are involved in forwarding the message in order to find a route to a destination. In our proposed modification, we can have the same outcome by using only a percentage of the number of the intermediate nodes. By using a lower probability than 1.0 , we use only a selected number of nodes to forward the messages thus reducing energy consumption and the number of retransmissions.
The following lines describe the proposed modification to the RREQ mechanism:

- Assume that there are $N$ nodes in the network and $n$ is the number of nodes in the neighbourhood of a transmitting node;

- In normal AODV route message forwarding, if a node is forwarding a packet then all the $n$ neighbours will try to forward the message again. If $P_{r x}$ is the power consumed by a node when receiving a route control message and $P_{t x}$ is the power consumed when a node forwards a route control message, then all $\mathrm{n}$ nodes will spend

$$
P_{s}=\left(P_{r x}+P_{t x}\right) * n
$$

- If $P_{i}$ is the sum of total initial power of the $n$ neighbouring nodes, then the total remaining power of the neighbouring node remaining is

$$
P_{\text {total }}=\left(P_{i}-P_{s}\right)
$$

- If probabilistic route message forwarding scheme is used, for example, assume that only $50 \%$ of the nodes are allowed to forward the message at any instance based on probability of 0.5 , this means that only $\frac{n}{2}$ nodes will receive and forward the message. This means that the network residual power will be

$$
P_{\text {total }}=\left(P_{i}-\frac{P_{s}}{2}\right)
$$

- If we compare Equations (2) and (3), the latter will preserve battery power and double the lifetime of the network whilst reducing the number of retransmissions.

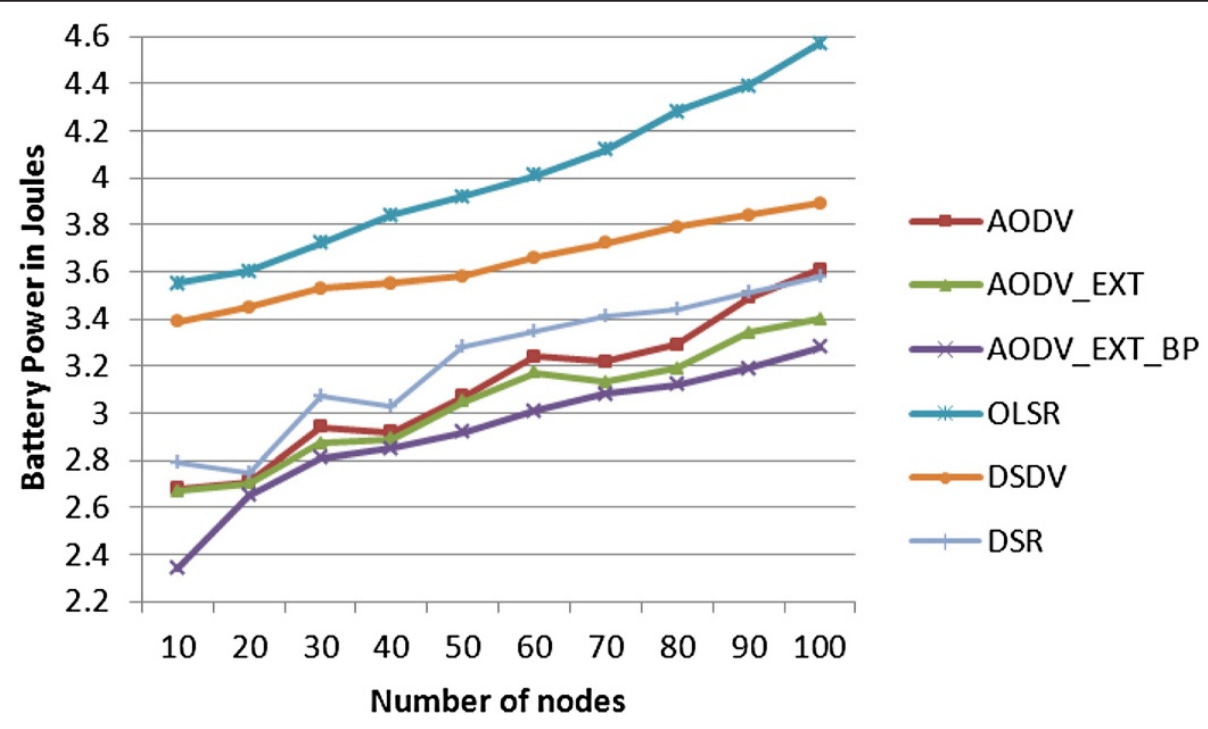

Figure 4 The average consumed power. 


\section{Simulation and metrics of a different message forwarding probability scheme on the RREQ mechanism of AODV protocol}

Network Simulator 2 (NS2) has been used to evaluate the modified AODV routing protocol using the probabilitybased message forwarding scheme (Table 1). The simulations were carried out to assess the performance of the routing protocols with network sizes of 10, 20, 30, 40, 50, $60,70,90$ and 100 nodes with mobile node speeds between 1 and $30 \mathrm{~m} / \mathrm{s}$. The mobility mode that has been used in all simulation was the random way point. In this model, nodes in a specific area choose some destination, and move there at a random speed uniformly chosen from $\left(0, V_{\max }\right)$, where $V_{\max }$ is the maximum speed of the simulation. The average speed is expected as the simulation evolves, and simulation results are in the form of an average over a period of time [23]. The simulation scenario has been repeated ten times and hence the results are the average of the ten times simulation scenarios. In all cases, the nodes send Constant Bit-rate (CBR) over user datagram protocol (UDP). The metrics that have been used to evaluate the performance of the network and protocols are the following [24]:

- Consumed power: the average consumed battery power.

- Number of packets dropped: this is the number of data packets that are not successfully sent to its destination.

- MAC Load: this is the ratio of the number of MAC layer messages propagated by every node in the network to the number of data packets successfully delivered to all destination nodes. In other words, the MAC Load is the average number of MAC messages generated for each data packet successfully delivered to the destination.

The simulation configuration and specification are specified in Table 2 .

Figure 1 shows that 0.3 (30\% of the intermediate nodes) and 0.4 ( $40 \%$ of the intermediate nodes) forward probability in terms of power consumption present very good results in every network size instead of using probability 1.0 which is the original probability that the AODV protocol uses. Moreover, good results are achieved by 0.2 probability (20\% of the intermediate nodes) but not in every network size. As we can see from the graph (Figure 1) in large-scale networks (more than 70 nodes), the use of 0.2 forward probability consumes more power than the 1.0 forward probability, meaning that needs more retransmissions in order to a valid route to the destination node to be found. By using less probability than $1.0(100 \%)$ we use only a selected number of nodes to forward the messages avoiding with this way the consumption of more energy.

Figure 2 shows that in small network sizes, smaller than 30 nodes, the forward probability-based scheme presents almost identical results, whilst in larger network sizes (bigger than 30 nodes) the use of 0.3 or 0.8 forwarding probability shows lower dropped packets than using probability $0.1,0.7$ and 1.0. This shows that when using of the following forwarding probabilities, 0.3 and 0.8 , the number of retransmissions is lower as the number of data packets that are successfully sent to their destinations are higher. Good results are also achieved with 0.5 forwarding probability but only for network sizes with the number of nodes between 70 and 100 .

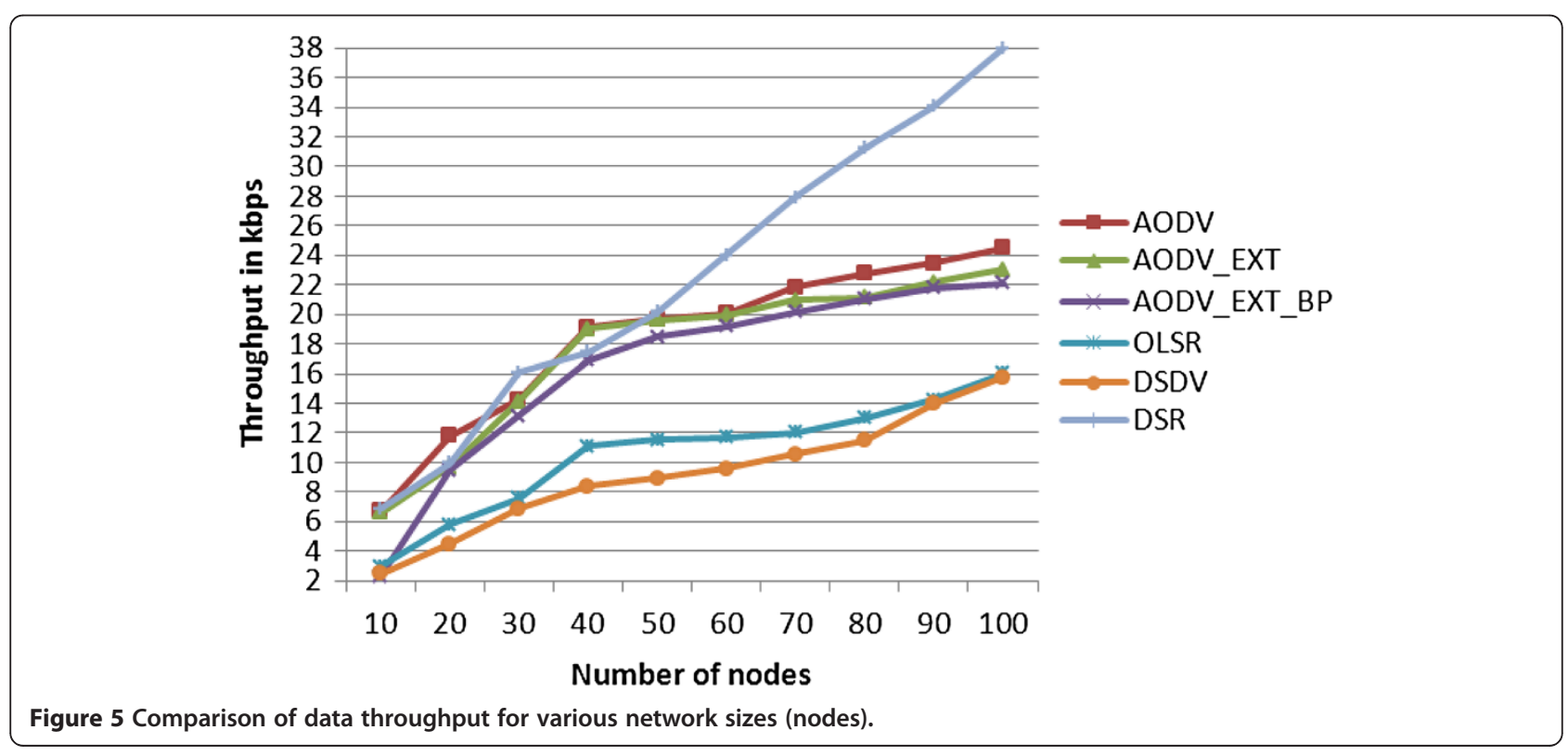


Figure 3 shows that the MAC Load increases rapidly when the probability is $0.1,0.7$ and 1.0 while on probability $0.3,0.4$ and 0.8 the MAC Load is very low. The MAC Load explains how successfully the number of data packets is delivered to the destination node. The forwarding probabilities 0.3 , 0.4 and 0.8 provide very low MAC Load meaning high success on data packet delivery while all the other forwarding probability values give very high MAC Load which lead to redundant retransmissions and depletion of battery power of the intermediate nodes.

All the above results have been used by the authors to develop a technique to change dynamically the forwarding probability according to the network density. In the following section, a heuristic approach using the Bayesian theory is presented.

\section{The modification of AODV RREQ mechanism using Bayesian probability approach}

Below are the steps that are followed during the RREQ and RREP processing mechanism of AODV:

$>$ When a source node, $S$, tries to send a packet to destination $D$

$>$ If $S$ does not know the next hop for $D$, then it broadcasts a route request message.

$>$ The RREQ message propagates in all directions to reach the destination $D$.

$>$ All the intermediate nodes that receive the RREQ message forward the packet to all its one hop neighbours.

$>$ If the destination, $D$, receives an RREQ message through a node $M$, then it sends an RREP to $S$ by forwarding it to $M$ since $M$ may contain at least one routing table entry for $S$.

$>$ On receiving the RREQ message through different nodes, the destination $D$ will send the RREP message through different nodes and they may reach the source node through different possible paths.

$>$ At the end, the source node $S$ will have different possible resolved paths to select from based on defined criteria.

In this RREQ mechanism, we intervene and use a version of the modified Bayesian probability theory in order to improve the function of the RREQ. In this section, the Bayesian probability theory and similar research that have been conducted by other researchers, together with a detailed explanation of our proposed algorithm, will be presented.

\section{Bayesian probability theory}

The Bayesian probability theory [25] manipulates conditional probabilities. The joint probability of two events, $A$ and $B$, can be expressed as

$$
\mathrm{P}(A B)=P(A / B) * P(B) \text { or } P(B / A) * P(A)
$$

In Bayesian probability theory, one of these events is called hypothesis and denoted with $H$ and the other is called data and denoted with $D$. The Bayesian probability theory assesses the probability of the observed data $D$ resulting from hypothesis $H$.

$$
P(H / D)=\frac{P(D / H) * P(H)}{P(D)}
$$

The term $P(H)$ is called the prior because it reflects prior knowledge before the data are considered. The term $P(D)$ is the result of the sum of $P(D \mid H) P(H)$ over all $H$. Finally, the term $P(H \mid D)$ is known as the posterior and reflects the probability of the hypothesis after consideration of the data.

There have been many efforts to us Bayesian probability in order to alleviate the flooding phenomenon. Jain et al. [26] proposed a heuristic algorithm with a route establishment technique using Bayesian approach. This algorithm improves the performance of route discovery by ameliorating the cost of route establishment using a history-based Bayesian method together with the relative region information of the destination node. The drawback of this effort is that it process and compares many information from each node (region, distance, status, destination node id and source node id) and which makes the algorithm complex, energy inefficient and time consuming in order to find the best route. Moreover, the simulations that have been conducted by other researchers were limited both in network size (number of nodes) and speed of the nodes. In large networks (bigger than 30 nodes), the behaviour of the network is different and there is bigger traffic congestion which causes unnecessary collision and packet overhead.

de Leoni et al. [27] have used the Bayesian probability theory in order to predict the disconnections that may occurred in MANETs. This research assumes that the nodes that are equipped with GPS receiver/transmitter and uses Bayesian filter to predict possible disconnections and control adjacent nodes to move to a location that that will provide continuous coverage. The disadvantage of the proposed algorithm is that it assumes that all nodes are equipped with GPS.

In our approach, we are using the Bayesian probability theory which is modified according to two major assumptions:

1. The posterior probability $P_{i}$ is the forward probability of five nodes which is $1(100 \%)$. In this case, when we have only five nodes or less the forward probability is $100 \%$.

2. The forward probability scheme $P_{i}$ depends on the minimum expected neighbours $(d)$ and the number of neighbours $(n)$. In this case, it multiplied the Bayesian probability $P_{i}$ by $1 / 2(n / d)$. 
Using this approach the RREQ mechanism was modified to broadcast only to a percentage of nodes for discovery the route. An example of how the Bayesian technique is implemented and how it affects the AODV protocol is described in details.

Assume that a node $D$ sends (forward) messages to anodes $D_{i}$ then using the probability forwarding scheme $P_{i}$ is

$$
P\left(D / D_{1}\right)=\frac{P_{\left(D / D_{1}\right) * \boldsymbol{P}_{(i)}}}{\left(D_{1} / D\right) * \boldsymbol{P}_{\left(D_{1}\right)}+\boldsymbol{P}_{\left(D_{2} / D\right)} \boldsymbol{P}_{* \boldsymbol{P}_{\left(D_{2}\right)}+\cdots+\boldsymbol{P}_{\left(D_{\mathrm{n}} / D\right) * \boldsymbol{P}_{\left(D_{n}\right)}}}}
$$

If it is assumed that there are nine nodes, in this case $P_{i}$ is the posterior probability which is the forwarding probability of five nodes which is 1 [when we have only five nodes or less the forward probability is 1 (100\%)]. The forward probability is calculating from the above equation:

$$
\begin{aligned}
P_{\left(D / D_{i}\right)} & =\frac{\frac{1}{2} * 1}{\frac{1}{2} * \frac{1}{2}+\frac{1}{2} * \frac{1}{2}+\frac{1}{2} * \frac{1}{2}+\frac{1}{2} * \frac{1}{2}+\frac{1}{2} * \frac{1}{2}+\frac{1}{2} * \frac{1}{2}+\frac{1}{2} * \frac{1}{2}+\frac{1}{2} * \frac{1}{2}+\frac{1}{2} * \frac{1}{2}} \\
& =\frac{\frac{1}{2} * 1}{\frac{9}{4}}=\frac{4}{18}=0.22
\end{aligned}
$$

This is the probability that if we forward the message with probability of $100 \%$ using the above approach $22 \%$ of the nodes will receive the message. This is for when $P(D 1 / D)=P(D 1)=\frac{1}{2}$ which is an independent probability that explains that the probability of the node receiving the message is $50 \%$ probability.

Table 3 presents the variables that have been used in the proposed algorithm.
If the RREQ is received from an intermediate node then there will be at least one possible route which includes that node in its route list. Therefore, if only selected nodes are allowed to forward the RREQ packet, then only these nodes will be included in the path list. In this proposed scheme, the neighbourhood density of an intermediate node is considered as a criterion in RREQ forwarding decision at intermediate node. It denotes that if the number of nodes in the neighbourhood is high, then the Bayesian probability of any node transmitting will decrease and therefore reduces the transmission overhead. Random selection of nodes from the neighbourhood set increases the chances of full network coverage. Greater savings could be achieved by using a range-dependent technique to select nodes for transmission but this can only be achieved at the cost of greater complexity.

\section{Proposed AODV_EXT_BP algorithm}

$>$ Any node $F_{i} i=1,2, \ldots n$ receiving the RREQ message will process the packet as follows:

$>$ If the RREQ message originated from the node $S_{i}$ or received at destination node $D_{i}$ just process it in the normal way. If $F_{i}$ is not the $S_{i}$ or $D_{i}$ then it will be an intermediate node:

$>$ The node $F_{i}$ will resolve the neighbourhood density

$D_{i}$ The Bayesian Probability $P_{i}$ is:

$$
\begin{aligned}
P_{i}= & \frac{P\left(D \mid D_{i}\right) * P(i)}{P(D 1 \mid D) * P(D 1)+P(D 2 \mid D) * P(D 2)+\ldots+P\left(D_{n} \mid D\right) * P\left(D_{n}\right)} \\
& * 1 / 2(n / d)
\end{aligned}
$$

$>$ If $P_{i}<r$ then

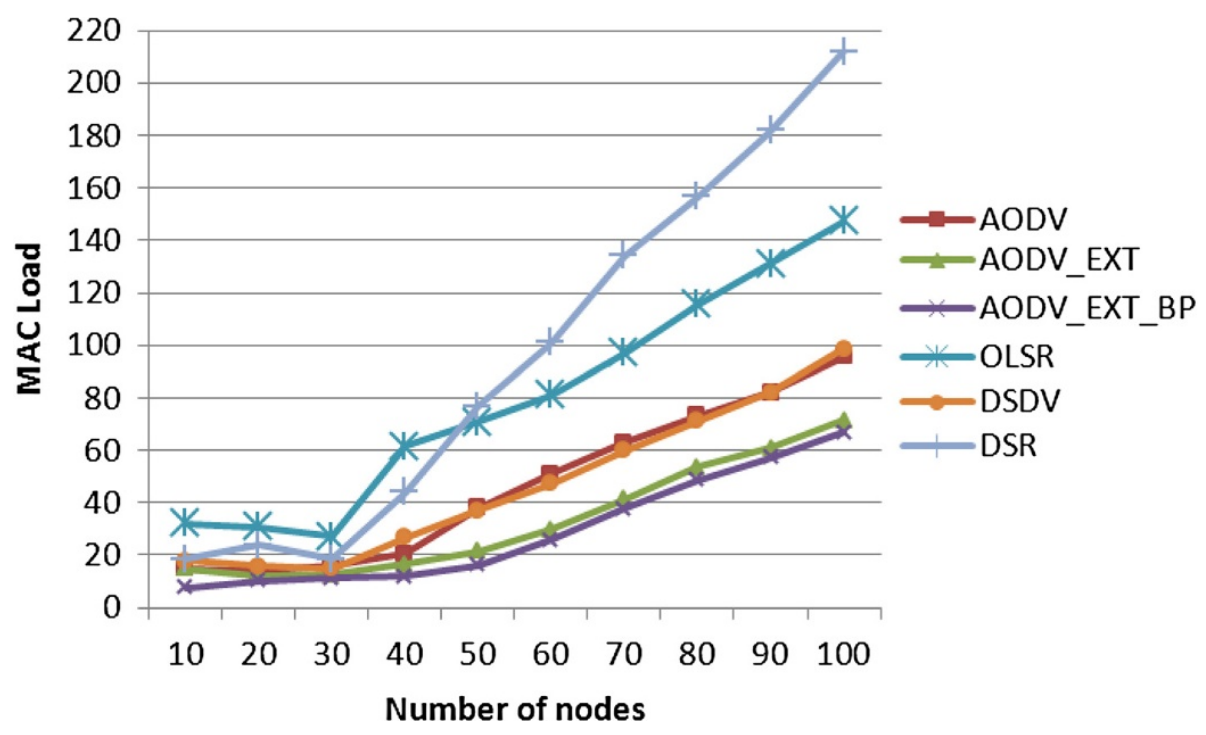

Figure 6 MAC Load against the number of nodes. 
Forward the RREQ message

Else

Ignore and drop the RREQ message

End

(Here $r$ is a random number between 0 and 1 )

The proposed algorithm is based on the Bayesian probability theory and disseminates the RREQ packet not at the whole network of nodes but only at those nodes that the algorithm chooses which is lesser than the complete network. In that case, the major differences are the following:

a. One of the main advantages of the proposed algorithm is that reduces the number of rebroadcasts without significantly compromising on its reachability, while the traditional probability scheme that the AODV uses, produces more re-broadcasts.

b. The Bayesian probability scheme forwards the RREQ packet into lessen nodes than the traditional probability scheme.

c. By using the Bayesian probability scheme the power consumption is less by $3.3 \%$ than the traditional probability scheme.

\section{Simulation and metrics of AODV RREQ mechanism using the Bayesian approach}

The NS2 has been used to evaluate the protocols [28-31]. The simulations were carried out to assess the performance of the routing protocols with network sizes of 10, 20, $30,40,50,60,70,90$ and 100 nodes with mobile node speeds between 1 and $30 \mathrm{~m} / \mathrm{s}$. The simulation scenario has been repeated ten times in order the procedure and the results to be reliable so all the values on each graph are the average of the ten times simulation scenarios. For simplicity, in all cases the nodes send CBR over UDP. The metrics that have been used to evaluate the performance of the network and protocols are the following [24]:

- Number of packets dropped: This is the number of data packets that are not successfully sent to its destination.

- Consumed power: The average consumed battery power.

- Throughput: This measures how well the network can constantly provide data to the sink. Throughput is the number of packet arriving at the sink per millisecond.

- MAC Load: This is the ratio of the number of MAC layer messages propagated by every node in the network to the number of data packets successfully delivered to all destination nodes. In other words, the MAC Load is the average number of MAC messages generated for each data packet successfully delivered to the destination.

- Control message overhead: This control message overhead is the total routing control messages transmitted and received in the network.

The simulation configuration and specification used are specified in Table 4.

\section{Results and discussions}

Figure 4 shows that AODV_EXT_BP consumes less power than the other five protocols. Most importantly, the power

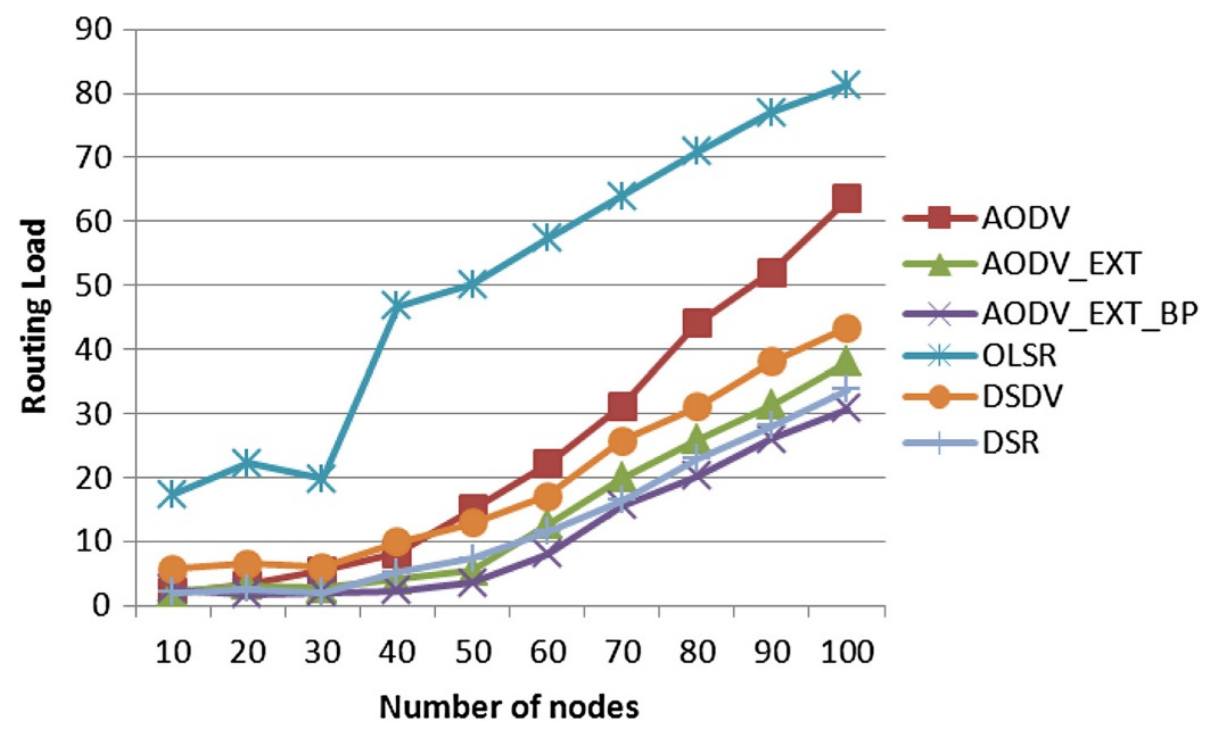

Figure 7 The control message overhead. 
consumption of AODV_EXT_BP-based network improves in comparison to that of already improved version of AODV the AODV_EXT and the standard AODV. The AODV_EXT_BP performances best in all the range of network densities. DSR protocol-based networks consumes more energy compared to AODV_EXT and AODV_EXT_BP but shows better performance in comparison to AODV when the number of nodes in the network is close to 100 nodes. On the contrary, the power consumption in networks using DSDV and OLSR rises steadily starting from fairly high levels. With increasing number of nodes, the energy depletion of OLSR-based networks increases faster than those for the other protocols. OLSR protocol uses a mechanism that constantly updates information about nodes in the neighbourhood and therefore consumes more energy. As the number of nodes in the network increases, more updates are required and hence proactive protocols perform poorly, especially when the network is subject to changes, e.g. in mobile environment.

Figure 5 shows the performance of the protocols based on data throughput. It shows that the traditional DSR achieves the best performance. However, AODV_EXT_BP shows very good performance in average density networks (until 50 nodes). With AODV_EXT_BP, AODV_EXT and AODV protocols, every node does not need to keep information regarding the route between two nodes. This reduces the amount of signalling required for route discovery and maintenance. OLSR and DSDV both show poor performances compared to the four protocols. This is because both are proactive protocols and require table updates and generate relatively high messaging overhead that can cause congestion in large networks, especially in mobile networks, and reduces data rate performance of the network. However, these protocols are better suited to low data rate transmission because their self-updating scheme ensures connectivity rather than the availability of bandwidth for application data. But in our simulation results the AODV_EXT_BP performs, also, as well as the other two routing protocols (OLSR, DSDV).

Figure 6 shows MAC loading of the protocols. It shows that for large networks, a relatively high number of messages are generated by OLSR and DSR-based networks. This explains why the number of successfully delivered data packets is very low and therefore increases re-transmissions. DSDV and AODV exhibit only moderate increases. The figure shows that the use of density-based scheme as applied in AODV_EXT_BP significantly reduces the number of routing messages in the network. The proposed scheme reduces the amount of messages retransmitted in every range of the network density; the improvement is $50 \%$ better than the standard AODV and by $3 \%$ over the AODV_EXT.

Figure 7 shows the routing control message overheads (routing load). In the case of AODV_EXT_BP, it is lower than that of the improved version of standard AODV, the AODV_EXT, and the original AODV protocol. DSR and DSDV show better performances due to the fact that they transmit and receive the least number of control messages. On the other hand, OLSR protocol has the worst performance of all the protocols and this degrades significantly as the number of nodes is between 30 and 40 .

Figure 8 shows the dropped packets in terms of network size. In case of AODV_EXT_BP, it is lower than that of the AODV_EXT and the original AODV protocol. DSDV presents almost the same results as AODV_EXT_BP because the mechanism that uses only adjacent nodes to send/receive packets has better performance in every scale of the network. OLSR and DSR show better performance in small-scale networks (until 30 nodes) after which both of them present low performances. The original AODV

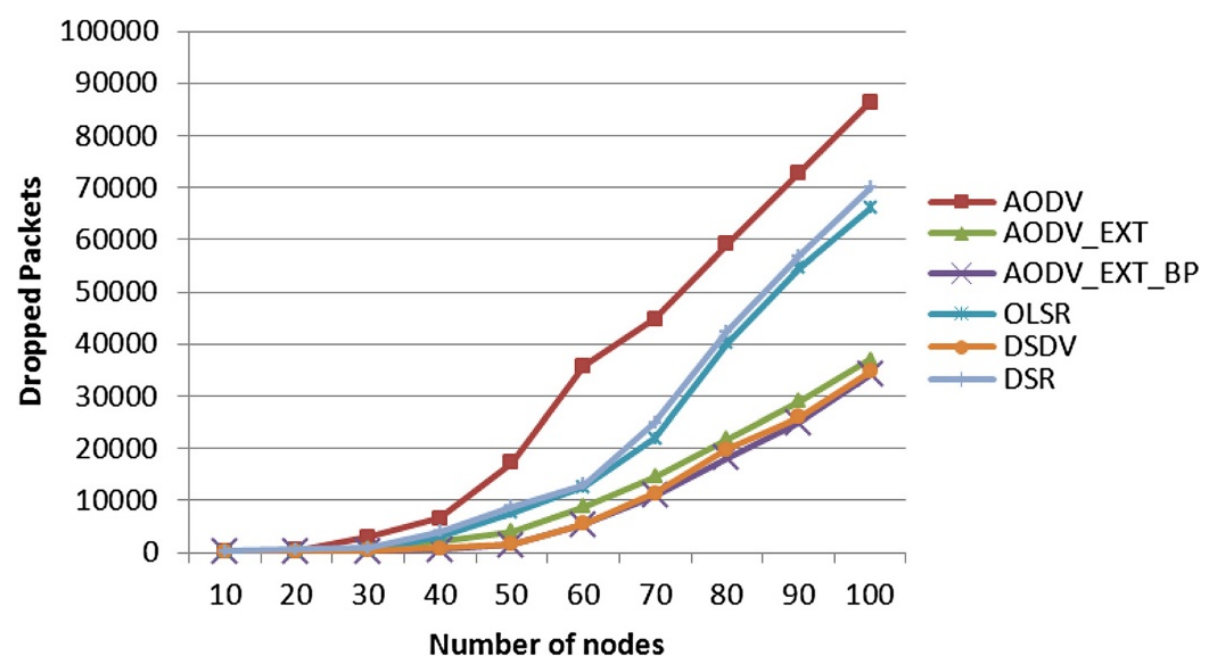

Figure 8 Dropped packets in terms of network size. 
protocol shows very poor performance of all the protocols when the number of nodes exceeds 20 .

\section{Conclusions}

The evaluation of six widely used protocols (AODV_EXT, AODV_EXT_BP, AODV, DSDV, DSR and OLSR) has been presented in this article. Their performances in different size networks and in mobile scenarios have been studied using simulations developed in NS2. AODV has been modified to use a modified Bayesian probabilistic approach for transmitting RREQ. The modified version has been named AODV_EXT_BP. Unlike in some probability-based approaches, where every node is assigned a fixed probability that does not ensure full network coverage, the technique proposed in this article combines concepts from maximum range node selection with node pruning to reduce redundant retransmissions in route request but offer connectivity and better network coverage guarantees inherent in deterministic techniques.

The reduction in route request transmissions in a network using AODV_EXT_BP has resulted in 3.3\% which is 0.3\% better energy efficiency savings compared to AODV_EXT [12], more than $70 \%$ reduction in the number of dropped packets because of reduced packet collision and increased data throughput. The results from this simulation can be compared with those in the research conducted by Khelifa and Maaza [32] proposed the Energy Reversed Ad-Hoc OnDemand Distance Vector (ER-AODV) routing protocol and consumes up to $2.0 \%$ more power than AODV_EXT_BP. Moreover, AODV_EXT_BP improves the data throughput by more than 20\% compared to the standard AODV and 12\% more than ER-AODV. The results also show that proactive protocols, whilst they are more reliable in terms of connectivity, exhibit poor performance in large networks. Reactive protocols, on the other hand, are more suited to large networks. Both classes of protocols perform poorly in large mobile networks due to large overheads associated with routing as the nodes move. A hybrid protocol such as AODV offers a compromise and the technique proposed in this article to reduce redundant re-transmissions based on transmitting node neighbourhood density has produced very promising results when compared to standard protocols. This research has showed that fine tuning of protocols to suit specific applications or traffic scenarios to achieve optimum performance in ad-hoc networks is essential.

In the future, a different probability approach such as a Monte Carlo algorithm implementation at the route request discovery mechanism, it could be challenging research, investigates whether it alleviates the storm phenomenon or not.

\section{Competing interests}

The authors declare that they have no competing interests.
Received: 17 February 2012 Accepted: 12 September 2012

Published: 23 October 2012

\section{References}

1. R Min, A Chandrakasan, Top five myths about the energy consumption of wireless communication. Mob. Comput. Commun. Rev. 7(1), 65-67 (2003)

2. V Kanakaris, D Ndzi, D Azzi, Ad-hoc networks energy consumption: a review of the ad-hoc routing protocols. J. Eng. Sci. Technol. Rev. 3(1), 162-167 (2010)

3. R Ramdhany, P Grace, G Coulson, D Hutchison, Dynamic deployment and reconfiguration of ad-hoc routing protocols. J. Internet Services Appl. $1(2), 135-152(2010)$

4. $Y X u$, J Heidemann, D Estrin, Geography-informed energy conservation for ad hoc routing, in Proceedings of the Seventh Annual ACM/IEEE International Conference on Mobile Computing and Networking, July 16-21 ((Rome) Italy, 2001), pp. 70-84

5. J Rabaey, J Ammer, JL da Silva Jr., D Patel, PicoRadio: ad-hoc wireless networking of ubiquitous low-energy sensor/monitor nodes, in Proceedings of the IEEE Computer Society Annual Workshop on VLSI (WVLSI'O0), April (Orlando, FL. USA, 2000), pp. 9-12

6. V Raghunathan, CL Pereira, MB Srivastava, RK Gupta, Energy aware wireless systems with adaptive power-fidelity tradeoffs. IEEE Trans. Very Large Scale Integr. (VLSI) Syst 13(2), 211-225 (2005)

7. CC Shen, C Srisathapornphat, R Liu, Z Huang, C Jaikaeo, E Lloyd, CLTC a cluster-based topology control framework for ad hoc networks. IEEE Trans. Mob. Comput. 3(1), 18-32 (2004)

8. M Bani Yassein, M Bani Khalaf, A Al-Dubai, A new probabilistic broadcasting scheme for mobile ad hoc on-demand distance vector (AODV) routed networks. J. Supercomput 53(1), 196-211 (2010)

9. M Bani Yassein, M Ould-Khaoua, LM Mackenzei, S Papanastasiou, Performance analysis of adjusted probabilistic broadcasting in mobile ad hoc networks. Int. J. Wirel. Inf. Netw 13(2), 127-140 (2006)

10. Y Sasson, D Cavin, A Schiper, Probabilistic broadcast for flooding in wireless mobile ad hoc networks, in Proceedings of IEEE wireless communication and networking conference (WCNC 2003), (2),March ( New Orleans, LA, USA, 2003), pp. 1124-1130

11. Q Zhang, DP Agrawal, Dynamic probabilistic broadcasting in MANETs. J. Parallel Distrib. Comput. 65(2), 220-233 (2005)

12. V Kanakaris, DL Ndzi, K Ovaliadis, Improving AODV performance using dynamic density driven route request forwarding. Int. J. Wirel. Mob. Netw. (IJWMN) 3(3), 16-27 (2011)

13. Survey: broadcasting in wireless sensor network, ad-hoc network and delaytolerant network. (2011). http://www.cs.tamu.edu/academics/tr/2011-9-2

14. N Karthikeyan, V Palanisamy, K Duraiswamy, Optimum density based model for probabilistic flooding protocol in mobile ad-hoc network. Eur. J. Sci. Res. 39(4), 577-588 (2010)

15. J Wu, F Dai, Efficient broadcasting with guaranteed coverage in mobile ad-hoc networks. IEEE Trans. Mob. Comput. 4(3), 259-270 (2005)

16. A Jamal, Probabilistic route discovery for wireless mobile ad-hoc networks (MANETs), PhD thesis (University of Glasgow, 2009)

17. JC Requena, T Vadar, R Kantola, N Beijar, AODV-OLSR scalable ad hoc routing proposal, in Symposium on Wireless Pervasive Computing ( Espoo, Finland, 2006), pp. 1-5. Jan

18. AH Abd Rahman, ZA Zukarnain, Performance comparison of AODV, DSDV and I-DSDV routing protocols in mobile ad-hoc networks. Eur. J. Sci. Res 31(4), 556-576 (2009)

19. The dynamic source routing protocol for mobile ad-hoc networks. http:// tools.ietf.org/html/draft-ietf-manet-dsr-10, Accessed 2 April 2008

20. F De Rango, M Fotino, S Marano, EE-OLSR: energy efficient OLSR routing protocol for mobile ad-hoc networks, in IEEE Military Communications Conference (2008), pp. 1-7

21. A Qayyum, L Viennot, A Laouiti, Multipoint relaying for flooding broadcast messages in mobile wireless networks (35th Annual Hawaii International Conference on System Sciences (HICSS'02), Hawai, 2002), pp. 3866-3875. (9),Jan

22. Ad-hoc on-demand distance vector (AODV) routing. (2000). http://tools.ietf org/html/draft-ietf-manet-aodv-08

23. J Yoon, M Liu, B Noble, Random waypoint considered harmful, in TwentySecond Annual Joint Conference of the IEEE Computer and Communications (INFOCOM 2003) (Ann Arbor, MI, USA, 2003), pp. 1312-1321. (2), March 
24. A Rahman, S Islam, A Talevski, Performance measurement of various routing protocol in ad-Hoc network, Proceedings of the International MultiConference of Engineers and Computer Scientists 2009 (IMECS), (1) ( Hong-Kong, 2009), pp. 321-323

25. Bayesian probability. (2010). http://people.cs.kuleuven.be/ danny. deschreye/urks-slides2007-1x3.pdf, Accessed 5 Jan

26. R Jain, M Parameswaran, C Hota, An efficient on-demand routing protocol for MANETs using Bayesian approach, in Third International Conference on Communication Systems and Networks (COMSNETS), Jan (Bangalore, India, 2011), pp. 1-4

27. M de Leoni, S Humayoun, M Mecella, R Russo, A Bayesian approach for disconnection management in mobile ad hoc networks, in 16th IEEE International Workshops on Enabling Technologies: Infrastructure for Collaborative Enterprises (WETICE 2007), June (Paris France, 2007)

28. Ns-2 network simulator. http://www.isi.edu/nsnam/ns/, Accessed 2 May 2008

29. CMU Monarch BPensions to NS-2. http://www.monarch.cs.cmu.edu/cmu-ns. html, Accessed 2 May 2008

30. Marc Greis' Tutorial for the UCB/LBNLNINT Network Simulator "ns". http://web. uct.ac.za/depts/commnetwork/tutorial_ns_full.pdf, Accessed 2 May 2008

31. Installing OLSR on NS-2. http://masimum.inf.um.es/um-olsr/html, Accessed 20 May 2008

32. S Khelifa, M Maaza, An energy multi-path AODV routing protocol in ad hoc mobile networks, in 5th International Symposium on IN Communications and Mobile Network (ISVC), Oct ( Oran Algeria, 2010), pp. 1-4

doi:10.1186/1687-1499-2012-318

Cite this article as: Kanakaris et al:: A new RREQ message forwarding

technique based on Bayesian probability theory. EURASIP Journal on

Wireless Communications and Networking 2012 2012:318.

\section{Submit your manuscript to a SpringerOpen ${ }^{\circ}$ journal and benefit from:}

- Convenient online submission

- Rigorous peer review

- Immediate publication on acceptance

- Open access: articles freely available online

- High visibility within the field

- Retaining the copyright to your article

Submit your next manuscript at $>$ springeropen.com 